\title{
INDUCED REPRESENTATIONS OF LIE ALGEBRAS AND A THEOREM OF BOREL-WEIL
}

\author{
BY \\ NOLAN R. WALLACH
}

1. Introduction. Let $\mathfrak{g}$ be a semisimple Lie algebra over $\boldsymbol{C}$ the complex numbers. Let $\mathfrak{h}$ be a Cartan subalgebra for $\mathfrak{g}$ and let $\Delta$ be the root system of $\mathfrak{g}$ with respect to $\mathfrak{h}$. We assume that $\Delta$ is linearly ordered. If $\mathfrak{n}^{+}\left(\right.$resp. $\left.\mathfrak{n}^{-}\right)$is the sum of the positive (resp. negative) root spaces of $\mathfrak{g}$ with respect to $\mathfrak{h}$ then $\mathfrak{g}=\mathfrak{n}^{-} \oplus \mathfrak{h} \oplus \mathfrak{n}^{-}$ (vector space direct sum) where $\left[\mathfrak{h}, \mathfrak{n}^{ \pm}\right] \subset \mathfrak{n}^{ \pm}$and $\mathfrak{n}^{ \pm}$is nilpotent on every finitedimensional $\mathfrak{g}$ module. Many results in the representation theory of semisimple Lie algebras depend essentially on this decomposition. In this paper we study Lie algebras over arbitrary fields with similar decompositions (see Definition 2.1). We show how every finite-dimensional simple module for such Lie algebras can be "induced" from finite-dimensional simple modules for the analogue of $\mathfrak{h}$. (See Theorem 3.1.) This induction procedure gives a new method of realizing the simple finite-dimensional modules for semisimple Lie algebras.

In this paper we also study the Lie group analogue of this procedure. As an application of the results of this paper we give a new proof of a theorem of BorelWeil as stated in Bott [1].

The author would like to thank Professor G. Hochschild for several stimulating conversations on the material of this paper and for his criticisms of the first draft of this paper.

2. Lie algebras with decompositions. Let $\mathfrak{g}$ be a Lie algebra over a field $k$.

DEFINITION 2.1. $g$ is said to decompose if there are subalgebras $\mathfrak{n}_{1}, \mathfrak{n}_{2}$ and $\mathfrak{h}$ of $\mathfrak{g}$ so that $\mathfrak{g}=\mathfrak{n}_{1} \oplus \mathfrak{h} \oplus \mathfrak{n}_{2}$ (vector space direct sum) and $\left[\mathfrak{h}, \mathfrak{n}_{i}\right] \subset \mathfrak{n}_{i}$ for $i=1$, 2. If furthermore $\mathfrak{n}_{1}, \mathfrak{n}_{2}$ are nilpotent on every finite-dimensional $\mathfrak{g}$ module, then the decomposition $\mathfrak{g}=\mathfrak{n}_{1} \oplus \mathfrak{h} \oplus \mathfrak{n}_{2}$ is called triangular.

We suppose that $\mathfrak{g}$ has a decomposition $\mathfrak{g}=\mathfrak{n}_{1} \oplus \mathfrak{h} \oplus \mathfrak{n}_{2}$. We set $\mathfrak{f}=\mathfrak{n}_{1} \oplus \mathfrak{h}$. If $W$ is an $\mathfrak{h}$ module, we denote by $\hat{W}$ the $\mathfrak{l}$ module with space $W$ so that $\mathfrak{n}_{1} \cdot \hat{W}=0$. If $\mathfrak{u}$ is a Lie algebra, $V$ a $\mathfrak{u}$ module, and $\mathfrak{n}$ is a subalgebra of $\mathfrak{u}$, then we set $V^{\mathfrak{n}}=\{v \in V \mid \mathfrak{n} \cdot v=0\}$. With these notational conventions in mind we proceed.

Let $U(\mathrm{~g})$ (see [3] or [4] for definitions) be the universal enveloping algebra for $\mathfrak{g}$; we assume that $U(\mathfrak{f}), U(\mathfrak{h}), U\left(\mathfrak{n}_{i}\right), i=1,2$, are the universal enveloping algebras of $\mathfrak{f}, \mathfrak{h}$ and $\mathfrak{n}_{\mathfrak{i}}, i=1,2$, imbedded (canonically) in $U(\mathfrak{g})$. The Poincaré-Birkhoff-Witt theorem implies that $U(\mathfrak{g})=U(\mathfrak{f}) \oplus U(\mathfrak{l}) \mathfrak{n}_{2} U\left(\mathfrak{n}_{2}\right)$ a left $U(\mathfrak{t})$, right $U(\mathfrak{h})$ module direct sum (abbreviated $(U(\mathfrak{l}), U(\mathfrak{h}))$ module direct sum). Let $\gamma$ be the projection

Received by the editors September 13, 1967. 
of $U(\mathfrak{g})$ onto $U(\mathfrak{f})$ associated with this decomposition. Then $\gamma$ is a $(U(\mathfrak{f}), U(\mathfrak{h}))$ module homomorphism.

We consider $U(\mathfrak{g})$ as a $(U(\mathfrak{f}), U(\mathfrak{g}))$ module. Thus if $W$ is a left $\mathfrak{h}$ module and if $\hat{W}$ is the associated $\mathfrak{k}$ module, then $\operatorname{Hom}_{U(\mathfrak{t})}(U(\mathfrak{g}), \hat{W})$ is naturally a left $U(\mathfrak{g})$ module. We define a map $\omega$ from $W$ to $\operatorname{Hom}_{U(t)}(U(\mathrm{~g}), \hat{W})$ by $\omega(v)(g)=\gamma(g) \cdot v$ where $v$ is looked upon as an element of $\hat{W}$ and $g \in U(\mathrm{~g})$. One sees easily that $\omega(v) \in \operatorname{Hom}_{U(\mathfrak{f})}(U(\mathrm{~g}), \hat{W})$. We also note that $\omega(h \cdot v)(g)=\gamma(g) h \cdot v=\gamma(g h) \cdot v=$ $\omega(v)(g \cdot h)=(h \cdot \omega(v))(g)$ for $h \in U(\mathfrak{h}), v \in W, g \in U(\mathfrak{g})$ and thus $\omega$ is a $U(\mathfrak{h})$ module homomorphism.

LEMMA 2.1. $\omega$ is an injection and $\omega(W)$ is the $U(\mathfrak{h})$ module direct summand $\operatorname{Hom}_{U(\mathfrak{t})}(U(\mathfrak{g}), \hat{W}) \circ \gamma$ of $\operatorname{Hom}_{U(\mathfrak{t})}(U(\mathfrak{g}), \hat{W})$.

Proof. If $f \in \operatorname{Hom}_{U(\mathfrak{t})}(U(\mathfrak{g}), \hat{W}) \circ \gamma$, then $f(a)=f(\gamma(a))=\gamma(a) f(1)=\omega(f(1))(a)$ for all $a \in U(\mathfrak{g})$. Thus $\omega(f(1))=f$. Furthermore if $\varepsilon$ is the evaluation map $\varepsilon(f)=f(1)$ for $f \in \operatorname{Hom}_{U(\mathfrak{t})}(U(\mathrm{~g}), \hat{W})$, then $\varepsilon \circ \omega=1_{W}$ which proves the lemma.

Let $W^{*}$ be the $U(\mathfrak{g})$ submodule of $\operatorname{Hom}_{U(\mathfrak{t})}(U(\mathfrak{g}), \hat{W})$ generated by $\omega(W)$, that is $W^{*}=U(\mathfrak{g}) \cdot \omega(W)$. Let $W_{1}, W_{2}$ be left $\mathfrak{h}$ modules and let $\varphi: W_{1} \rightarrow W_{2}$ be an $\mathfrak{h}$ module homomorphism. We regard $\varphi$ as a $\mathfrak{t}$ module homomorphism of $\hat{W}_{1}$ to $\hat{W}_{2} . \varphi$ induces a $U(\mathfrak{g})$ module homomorphism $\varphi^{*}: \operatorname{Hom}_{U(\mathfrak{f})}\left(U(\mathfrak{g}), \hat{W}_{1}\right) \rightarrow$ $\operatorname{Hom}_{U(\mathfrak{f})}\left(U(\mathrm{~g}), \hat{W}_{2}\right)$ by $\varphi^{*}(f)=\varphi \circ f . \varphi^{*}$ commutes with the map $f \rightarrow f \circ \gamma$ and thus $\varphi^{*}\left(W_{1}^{*}\right) \subset W_{2}^{*}$. Thus $W \rightarrow W^{*}, \varphi \rightarrow \varphi^{*}$ is a functor from the category of left $\mathfrak{h}$ modules to the category of left $\mathrm{g}$ modules.

We make the observation

Lemma 2.2. $\operatorname{Hom}_{U(\mathfrak{f})}(U(\mathfrak{g}), \hat{W}) \circ \gamma=\operatorname{Hom}_{U(\mathfrak{f})}(U(\mathfrak{g}), \hat{W})^{\mathfrak{n}_{2}}$.

Proof. If $y \in \mathfrak{n}_{2}$, then $(y \cdot(f \circ \gamma))(a)=f(\gamma(a y))=f(0)=0$ for all $a \in U(\mathrm{~g})$. Conversely if $y \cdot f=0$ for all $y \in \mathfrak{n}_{2}$, then $f\left(U(\mathfrak{t}) \mathfrak{n}_{2} U\left(\mathfrak{n}_{2}\right)\right)=0$; hence $f=f \circ \gamma$.

We also note

LEMMA 2.3. Let $W_{1}$ and $W_{2}$ be left $\mathfrak{h}$ modules. $W_{1}$ and $W_{2}$ are isomorphic $\mathfrak{h}$ modules if and only if $W_{1}^{*}$ and $W_{2}^{*}$ are isomorphic $\mathrm{g}$ modules.

Proof. If $\varphi: W_{1} \rightarrow W_{2}$ is an onto isomorphism, then clearly so is $\varphi^{*}: W_{1}^{*} \rightarrow W_{2}^{*}$. Suppose that $\varphi^{*}: W_{1}^{*} \rightarrow W_{2}^{*}$ is an onto $U(\mathrm{~g})$ isomorphism. Then $\varphi^{*}: W_{1}^{* n_{2}} \rightarrow W_{2}^{* n_{2}}$ is an $\mathfrak{h}$ module isomorphism. Hence $\varphi^{*}: \omega\left(W_{1}\right) \rightarrow \omega\left(W_{2}\right)$ is an $\mathfrak{h}$ module isomorphism and hence $\varphi^{*}$ induces an $\mathfrak{h}$ module isomorphism $\psi$ of $W_{1}$ onto $W_{2}$ so that $\psi^{*}=\varphi^{*}$.

3. Lie algebras with triangular decompositions. In this section we continue the notation of $\S 2$. We show that if $\mathfrak{g}=\mathfrak{n}_{1} \oplus \mathfrak{h} \oplus \mathfrak{n}_{2}$ is a decomposition for a Lie algebra $\mathfrak{g}$ then simple $\mathfrak{g}$ modules satisfying certain conditions can be "induced" as in $\S 2$. In particular we will show that if $\mathfrak{n}_{1} \oplus \mathfrak{h} \oplus \mathfrak{n}_{2}$ is a triangular decomposition then every finite-dimensional simple $g$ module can be induced. 
Let $V$ be a left $g$ module. Let $\tilde{V}$ be the (right) dual module. Let (as usual) $\tilde{V}^{\mathfrak{n}_{1}}=\left\{\lambda \in \tilde{V} \mid \lambda \cdot \mathfrak{n}_{1}=0\right\}$. Then $\tilde{V}^{\mathfrak{n}_{1}}$ is an $\mathfrak{h}$ module and $\tilde{V}^{\mathfrak{n}_{1}} \cdot \mathfrak{n}_{1}=0$ hence $\tilde{V}^{\mathfrak{n}_{1}}$ is a $\mathfrak{t}$ module and clearly $\left(\tilde{V}^{\mathfrak{n}_{1}}\right)^{\wedge}=\widetilde{V}^{\mathfrak{n}_{1}}$. Now $\left(\tilde{V}^{\mathfrak{n}_{1}}\right)^{\sim}$ is a left $\mathfrak{t}$ module. For each $v \in V$ we define a mapping $\alpha(v): U(\mathfrak{g}) \rightarrow\left(\tilde{V}^{\mathfrak{n}_{1}}\right)^{\sim}$ by $\alpha(v)(a)(\lambda)=\lambda(a \cdot v)$ for all $a \in U(\mathfrak{g})$, $\lambda \in \tilde{V}^{\mathfrak{n}_{1}}$.

Proposition 3.1. If $V$ is a simple left $\mathrm{g}$ module and if $\tilde{V}^{\mathfrak{n}_{1}} \neq\{0\}$, then $\alpha$ is an injection of the $\mathrm{g}$ module $V$ into the $\mathrm{g}$ module $\operatorname{Hom}_{U(\mathfrak{t})}\left(U(\mathrm{~g}),\left(\tilde{V}^{\mathfrak{n}_{1}}\right)^{\sim}\right)$.

Proof. Let $a \in U(\mathfrak{f}), \quad b \in U(\mathfrak{g}), \quad v \in V, \lambda \in \widetilde{V}^{\mathfrak{n}_{1}}$, then $(\alpha(v)(a \cdot b))(\lambda)=\lambda(a \cdot b \cdot v)$ $=(\lambda \cdot a)(b \cdot v)=(\alpha(v)(b))(\lambda \cdot a)=(a \cdot(\alpha(v)(b)))(\lambda)$. Thus $\alpha(v)(a \cdot b)=a \cdot(\alpha(v)(b))$ and thus $\alpha(v) \in \operatorname{Hom}_{U(\mathfrak{f})}\left(U(\mathfrak{g}),\left(\tilde{V}^{\mathfrak{n}_{1}}\right)^{\sim}\right)$. We next show that $\alpha$ is a $U(\mathfrak{g})$ module homomorphism. Let $a, b \in U(\mathfrak{g}), \quad v \in V$, and $\lambda \in \tilde{V}^{\mathfrak{n}_{1}}$. Then $(\alpha(a \cdot v))(b)(\lambda)=\lambda(b \cdot a \cdot v)$ $=\alpha(v)(b \cdot a)(\lambda)=(a \cdot \alpha(v))(b)(\lambda)$. Hence $\alpha(a \cdot v)=a \cdot \alpha(v)$ as asserted. Since $\alpha \neq 0$ and $V$ is simple we find that $\alpha$ is an injection of $g$ modules.

Proposition 3.2. Let $W$ be an $\mathfrak{h}$ module. Let $V$ be a nonzero $\mathfrak{g}$ submodule of $\operatorname{Hom}_{U, \mathfrak{t}}(U(\mathfrak{g}), \hat{W})$ such that $V^{\mathfrak{n}_{2}} \neq\{0\}$; then $V \cap W^{*} \neq\{0\}$. If furthermore $W$ is simple, then $V \supset W^{*}$.

Proof. $V^{\mathfrak{n}_{2}} \subset \operatorname{Hom}_{U(\mathfrak{t})}(U(\mathfrak{g}), \hat{W})^{\mathfrak{n}_{2}}=\operatorname{Hom}_{U(\mathfrak{t})}(U(\mathfrak{g}), \hat{W}) \circ \gamma=\omega(W)$ by Lemmas 2.1 and 2.2. Thus $0 \neq U(\mathfrak{g}) \cdot V^{\mathfrak{n}_{2}} \subset W^{*}$ and hence $V \cap W^{*} \neq\{0\}$. If $W$ is a simple $\mathfrak{h}$ module, then $U(\mathfrak{h}) \cdot V^{\mathfrak{n}_{2}}=\omega(W)$ and thus $V \supset U(\mathfrak{g}) \cdot \omega(W)=W^{*}$.

We now assume that $\mathfrak{g}=\mathfrak{n}_{1} \oplus \mathfrak{h} \oplus \mathfrak{n}_{2}$ is a triangular decomposition. We note that if one wishes more "general" results than the following, one can hypothesize just enough nilpotence on $\mathfrak{n}_{1}$ and $\mathfrak{n}_{2}$ to make the following proofs "go through".

Lemma 3.1. Let $V$ be a simple finite-dimensional $\mathfrak{g}$ module. Then $\left(\mathfrak{n}_{1} \cdot V\right)^{\mathfrak{n}_{2}}=\{0\}$.

Proof. Suppose that $\left(\mathfrak{n}_{1} \cdot V\right)^{\mathfrak{n}_{2}} \neq\{0\}$. Then $V=U(\mathfrak{g})\left(\mathfrak{n}_{1} \cdot V\right)^{\mathfrak{n}_{2}}$ since $V$ is simple. $U(\mathfrak{t}) \mathfrak{n}_{2} U\left(\mathfrak{n}_{2}\right) \cdot\left(\mathfrak{n}_{1} \cdot V\right)^{\mathfrak{n}_{2}}=\{0\} \quad$ implies that $V=U(\mathfrak{l}) \cdot\left(\mathfrak{n}_{1} \cdot V\right)^{\mathfrak{n}_{2}} . \quad U(\mathfrak{h})\left(\mathfrak{n}_{1} \cdot V\right)^{\mathfrak{n}_{2}}$ $\subset\left(\mathfrak{n}_{1} \cdot V\right)^{\mathfrak{n}_{2}}$. Hence $V=U\left(\mathfrak{n}_{1}\right)\left(\mathfrak{n}_{1} \cdot V\right)^{\mathfrak{n}_{2}} \subset U\left(\mathfrak{n}_{1}\right) \cdot \mathfrak{n}_{1} \cdot V$. Since $\mathfrak{n}_{1}$ acts nilpotently on $V$ this implies $V=\{0\}$ which is a contradiction.

Proposition 3.3. Let $\mathfrak{g}=\mathfrak{n}_{1} \oplus \mathfrak{h} \oplus \mathfrak{n}_{2}$ be a triangular decomposition for $\mathfrak{g}$. If $V$ is a finite-dimensional simple $\mathrm{g}$ module, then $V$ is $\mathrm{g}$ isomorphic to $\left(V^{\mathfrak{n}_{2}}\right)^{*}$ and $V^{\mathfrak{n}_{2}}$ is a simple $\mathfrak{h}$ module.

Proof. We use the notation of Proposition 3.1. Let $\iota: V^{\mathfrak{n}_{2}} \rightarrow\left(\tilde{V}^{\mathfrak{n}_{1}}\right)^{\sim}$ be given by $\iota(v)(\lambda)=\lambda(v)$ for each $\lambda \in \tilde{V}^{\mathfrak{n}_{1}}, v \in V^{\mathfrak{n}_{2}}$. Then if $a \in U(\mathfrak{h})$ we have $\iota(a v)(\lambda)=\lambda(a v)$ $=(\lambda \cdot a)(v)=(a \cdot \iota(v))(\lambda)$ and thus $\iota$ is a $U(\mathfrak{h})$ module homomorphism. We look at $\iota$ as a $U(\mathfrak{f})$ module homomorphism of $\left(V^{\mathfrak{n}_{2}}\right)^{\wedge}$ to $\left(\tilde{V}^{\mathfrak{n}_{1}}\right)^{\sim}$. Ker $\iota=\left(\mathfrak{n}_{1} \cdot V\right)^{\mathfrak{n}_{2}}=\{0\}$ by Lemma 3.1. Hence $\left(V^{\mathfrak{n}_{2}}\right)^{\wedge}$ is isomorphic with $\left(\tilde{V}^{\mathfrak{n}_{1}}\right)^{\sim}$. Now if $v \in V^{\mathfrak{n}_{2}}, g \in U(\mathfrak{g})$, $\lambda \in \tilde{V}^{\mathfrak{n}_{1}}$ then $\alpha(v)(g)(\lambda)=\lambda(g \cdot v)=\lambda(\gamma(g) \cdot v)=\iota(\gamma(g) \cdot v)(\lambda)=(\gamma(g) \cdot \iota(v))(\lambda)$ (where $\gamma$ is as in the beginning of $\S 2$ and $v$ is looked upon as an element of $\left.\left(V^{\mathfrak{n}_{2}}\right)^{\wedge}\right)$. Thus $\alpha\left(V^{\mathfrak{n}_{2}}\right)=\omega\left(\iota\left(V^{\mathfrak{n}_{2}}\right)\right)$ in $\operatorname{Hom}_{U(\mathfrak{f})}\left(U(\mathfrak{g}), \iota\left(\left(V^{\mathfrak{n}_{2}}\right)^{\wedge}\right)\right) \subset \operatorname{Hom}_{U(\mathfrak{t})}\left(U(\mathfrak{g}),\left(\tilde{V}^{\mathfrak{n}_{1}}\right)^{\sim}\right)$. Thus $\alpha(V)$ 
$\supset\left(\iota\left(V^{\mathfrak{n}_{2}}\right)\right)^{*}$. Since $\alpha(V)$ is simple this implies $\alpha(V)=\left(\iota\left(V^{\mathfrak{n}_{2}}\right)\right)^{*}$. Since $\iota$ is an isomorphism this implies that $V$ is isomorphic to $\left(V^{\mathfrak{n}_{2}}\right)^{*}$ (Lemma 2.3). Suppose $V^{\mathfrak{n}_{2}}$ is not a simple $\mathfrak{h}$ module. Let $W$ be a nonzero $\mathfrak{h}$ submodule of $V^{\mathfrak{n}_{2}}$. Then $\operatorname{Hom}_{U(\mathfrak{f})}(U(\mathfrak{g}), \hat{W}) \subset \operatorname{Hom}_{U(\mathfrak{t})}\left(U(\mathfrak{g}),\left(V^{\mathfrak{n}_{2}}\right)^{\wedge}\right)$ and thus $W^{*} \subset\left(V^{\mathfrak{n}_{2}}\right)^{*}$. Since $\left(V^{\mathfrak{n}_{2}}\right)^{*}$ is simple this implies that $W^{*}=\left(V^{\mathfrak{n}_{2}}\right)^{*}$. Thus $W^{* \mathfrak{n}_{2}}$ is isomorphic with $V^{\mathfrak{n}_{2}}$. This implies that $W$ is isomorphic with $V^{\mathfrak{n}_{2}}$ and thus $W=V^{\mathfrak{n}_{2}}$.

We recapitulate the results of this section with

THEOREM 3.1. Let $\mathfrak{g}=\mathfrak{n}_{1} \oplus \mathfrak{h} \oplus \mathfrak{n}_{2}$ be a triangular decomposition for the Lie algebra $\mathrm{g}$.

(a) If $W$ is a simple $\mathfrak{h}$ module, then $\operatorname{Hom}_{U(\mathfrak{t})}(U(\mathfrak{g}), \hat{W})$ contains at most one nonzero finite-dimensional simple $\mathfrak{g}$ module. Such a nonzero finite-dimensional simple $\mathrm{g}$ module exists if and only if $\operatorname{dim} W^{*}<\infty$. If $\operatorname{dim} W^{*}<\infty$, then $W^{*}$ is the finitedimensional nonzero simple $\mathrm{g}$ module in $\operatorname{Hom}_{U(\mathfrak{t})}(U(\mathfrak{g}), \hat{W})$.

(b) If $V$ is a finite-dimensional simple $\mathfrak{g}$ module, then $V^{\mathfrak{n}_{2}}$ is a simple $\mathfrak{h}$ module and $V$ is $\mathrm{g}$ isomorphic with $\left(V^{\mathfrak{n}_{2}}\right)^{*}$.

Proof. We must only prove (a). Let $V$ be a nonzero finite-dimensional simple submodule of $\operatorname{Hom}_{U(\mathfrak{t})}(U(\mathfrak{g}), \hat{W})$; then by Proposition 3.2,Vつ $W^{*}$. Hence by simplicity $V=W^{*}$ if and only if $\operatorname{dim} W^{*}<\infty$ and the existence of $V$ is impossible if $\operatorname{dim} W^{*}=\infty$.

The technique of "double dualization" used in this section is due to Godement (see Želobenko [2]).

4. Lie groups with triangular decompositions. In this section we assume that $k=\boldsymbol{R}$ (the real numbers) or $\boldsymbol{C}$ (the complex numbers). By $k$-analytic we will mean analytic for $k=\boldsymbol{R}$, holomorphic for $k=\boldsymbol{C}$.

Definition 4.1. Let $G$ be a $k$-Lie group with Lie algebra $\mathfrak{g}$. If $\mathfrak{g}=\mathfrak{n}_{1} \oplus \mathfrak{h} \oplus \mathfrak{n}_{2}$ is a triangular decomposition for $\mathfrak{g}$ and if $N_{1}, H, N_{2}$ are respectively the connected subgroups of $G$ corresponding to $\mathfrak{n}_{1}, \mathfrak{h}$ and $\mathfrak{n}_{2}$, then $G$ is said to have a triangular decomposition if $N_{1} \cdot H$ is closed in $G$.

Let $G$ be a $k$-Lie group with triangular decomposition $N_{1}, H, N_{2}$. Set $K=H \cdot N_{1}$. Let $W$ be a finite-dimensional $k$-analytic $H$ module. As before we let $\hat{W}$ be the $K$ module $W$ with $N_{1}$ acting as the identity. Let $\Gamma(W)$ be the $k$ vector space of all $k$-analytic functions $f: G \rightarrow \hat{W}$ such that $f(u \cdot g)=u \cdot f(g)$ for all $u \in K, g \in G$. $G$ acts on $\Gamma(W)$ on the left by $\left(g_{0} \cdot f\right)(g)=f\left(g g_{0}\right)$. Let $\Gamma_{0}(W)$ be the subspace of those elements $f \in \Gamma(W)$ such that $G \cdot f$ is contained in a finite-dimensional subspace of $\Gamma(W)$.

If $V$ is a finite-dimensional $k$ analytic left $G$ module, then we can define an action of $\mathfrak{g}$ on $V$ as follows: $x \cdot v=d e^{t x} \cdot v /\left.d t\right|_{t=0}$ for $x \in \mathfrak{g}, v \in V$. With this action of $\mathfrak{g}$ on $V$ we have $\exp (x) \cdot v=\sum x^{n} / n ! \cdot v$. If $G$ is a simply connected $k$-Lie group and if $V$ is a finite-dimensional $\mathrm{g}$ module, then $V$ is a $G$ module with the action of $G$ given locally by $\exp (x) \cdot v=\sum x^{n} / n ! \cdot v$ for all $x \in \mathfrak{g}, v \in V$. Thus we denote $V$ as a $G$ or $g$ module by the same symbol if $G$ is simply connected. 
If $W$ is a $k$-analytic $H$ module, then $W$ may be looked upon as an $\mathfrak{h}$ module as above. Furthermore $\Gamma(W)$ is a $g$ module under the action $(x \cdot f)(g)=d f\left(\dot{g} e^{t x}\right) /\left.d t\right|_{t=0}$. Thus we look upon $\Gamma(W)$ as both a $G$ and a $g$ module. With these conventions in mind we have

THEOREM 4.1. Let $G$ be a connected and simply connected $k$-Lie group with triangular decomposition $N_{1}, H, N_{2}$. Let $W$ be a finite-dimensional simple $k$-analytic $H$ module. If $\operatorname{dim} W^{*}=\infty$, then $\Gamma_{0}(W)=\{0\}$. If $\operatorname{dim} W^{*}<\infty$, then $\Gamma_{0}(W)$ contains $a G$ (hence g) module isomorphic with $W^{*}$.

Proof. Let $f \in \Gamma(W)$. We define $\tilde{f}: U(\mathfrak{g}) \rightarrow \hat{W}$ by $\tilde{f}(x)=(x \cdot f)(e)$ where $x \in U(\mathfrak{g})$ and $e$ is the unit of $G$. $\tilde{f}$ is clearly linear on $U(\mathfrak{g})$. If $u \in \mathfrak{f}=\mathfrak{n}_{1} \oplus \mathfrak{h}, x \in U(\mathfrak{g})$, then

$$
\begin{aligned}
\tilde{f}(u \cdot x) & =((u x) \cdot f)(e)=(u \cdot(x \cdot f))(e)=d(x \cdot f) e^{t u} /\left.d t\right|_{t=0} \\
& =d e^{t u} \cdot((x \cdot f)(e)) /\left.d t\right|_{t=0}=u \cdot(x \cdot f(e))=u \cdot \tilde{f}(x) .
\end{aligned}
$$

Thus $\tilde{f} \in \operatorname{Hom}_{U(\mathfrak{f})}(U(\mathfrak{g}), \hat{W})$. Let $A f=\tilde{f}$. By analyticity $A$ is one-to-one. If $g_{0} \in U(\mathfrak{g})$, $f \in \Gamma(W)$, then $A\left(g_{0} f\right)(g)=\left(g g_{0} \cdot f\right)(e)=\tilde{f}\left(g g_{0}\right)=\left(g_{0} \cdot A f\right)(g)$. Thus $A$ is a $U(g)$ module isomorphism. If $f \in \Gamma_{0}(W)$, then $U(\mathfrak{g}) \cdot A f$ is a finite-dimensional submodule of $\operatorname{Hom}_{U(\mathfrak{f})}(U(\mathfrak{g}), \hat{W})$. By Theorem 3.1 (a) $U(\mathfrak{g}) \cdot A f \supset W^{*}$ if $A f \neq 0$. Thus if $\operatorname{dim} W^{*}=\infty$ we must have $A f=0$ and hence $f=0$. If $\operatorname{dim} W^{*}<\infty$, then by Theorem 3.1 (a) $W^{*}$ is the only nonzero irreducible submodule of $\operatorname{Hom}_{U(t)}(U(\mathrm{~g}), \hat{W})$. Thus if $A f \neq 0$ for some $f \in \Gamma_{0}(W), U(\mathrm{~g}) \cdot A f \supset W^{*}$ and hence $A^{-1} \cdot W^{*}$ is a submodule of $\Gamma_{0}(W)$ isomorphic with $W^{*}$. Now if $r \in W^{*}$ we define a mapping $\hat{r}: G \rightarrow \hat{W}$ which is $k$-analytic by $\hat{r}(g)=(g \cdot r)(1)$. We note that

$$
\begin{aligned}
\hat{r}(\exp x g) & =(\exp (x) \cdot g \cdot r)(1)=\sum x^{n} / n ! \cdot(g \cdot r)(1)=\sum g \cdot r\left(x^{n} / n !\right) \\
& =\sum x^{n} / n ! \cdot(g \cdot r(1))=\exp (x) \cdot \hat{r}(g)
\end{aligned}
$$

for $x \in \mathfrak{f}, g \in G$. Thus $\hat{r} \in \Gamma_{0}(W),(\hat{r})^{\sim}=r$ and the theorem is proved.

In the case $G$ is semisimple we can strengthen Theorem 4.1.

Corollary to TheOREM 4.1. Let $G$ be a connected, simply connected, semisimple $k$-analytic Lie group with triangular decomposition $N_{1}, H, N_{2}$. Let $W$ be a simple finite-dimensional $k$-analytic $H$ module. If $\operatorname{dim} W^{*}<\infty$, then $\Gamma_{0}(W)$ is equivalent to $W^{*}$ as a $\mathfrak{g}$ (hence $\left.G\right)$ module.

Proof. Let $A$ be as in the proof of Theorem 4.1. Then $U(\mathrm{~g}) \cdot A f \supset W^{*}$ for each $f \in \Gamma_{0}(W), f \neq 0 . \operatorname{dim} U(\mathfrak{g}) \cdot A f<\infty$ implies that as a $\mathrm{g}$ module $U(\mathrm{~g}) \cdot A f$ is a direct sum of simple $g$ modules. Theorem 3.1 (a) asserts that $W^{*}$ is the only finitedimensional nonzero $\mathrm{g}$ module in $\operatorname{Hom}_{U(\mathfrak{t})}(U(\mathfrak{g}), \hat{W})$; thus $U(\mathfrak{g}) \cdot A f=W^{*}$. In particular, $A \cdot \Gamma_{0}(W) \subset W^{*}$ and hence $A \cdot \Gamma_{0}(W)=W^{*}$. $A$ is a $G$ module isomorphism; thus the corollary is proved.

\section{Examples and applications.}

EXAMPLE 1. Let $k$ be of characteristic 0 . If $g$ is a Lie algebra over $k$ and if $\mathfrak{g}=\mathfrak{n}_{1}+\mathfrak{h}+\mathfrak{n}_{2}$ is a decomposition for $\mathfrak{g}$ and if there are solvable subalgebras $\mathfrak{S}_{1}$ 
and $\mathfrak{S}_{2}$ of $\mathfrak{g}$ such that $\mathfrak{n}_{1} \subset\left[\mathfrak{s}_{i}, \mathfrak{F}_{i}\right]$ for $i=1,2$, then $\mathfrak{g}=\mathfrak{n}_{1}+\mathfrak{h}+\mathfrak{n}_{2}$ is a triangular decomposition. This follows directly from Lie's and Engel's Theorems.

Example 2. The Borel-Weil Theorem. We first recall the classification of compact, homogeneous, Kaehlerian, simply connected manifolds (see Wang [5]) which are called Kaehlerian $C$-spaces following Wang's terminology. Let $G$ be a complex connected, simply connected Lie group with Lie algebra $\mathfrak{g}$. Let $\tilde{\mathfrak{h}}$ be a Cartan subalgebra for $\mathfrak{g}$ and let $\Delta$ be the root system of $\mathfrak{g}$ with respect to $\tilde{\mathfrak{h}}$ (see [3] or [4] for definitions). Let $\pi$ be a fixed fundamental system for $\Delta$. Let $\pi_{1}$ be a subset of $\pi$. We set $\Delta\left(\pi_{1}\right)=\left\{\alpha \in \Delta \mid \alpha=\sum_{y \in \pi_{1}} \mathfrak{n}_{\gamma} \gamma, \mathfrak{n}_{\gamma}\right.$ an integer $\}$. Let $>$ be a lexicographic order on $\Delta$ determined by $\pi$. Set $\mathfrak{g}_{\alpha}=\{x \in \mathfrak{g} \mid[h, x]=\alpha(h) x$ for all $h \in \tilde{\mathfrak{h}}\}$, $\alpha \in \Delta$. We set $\Sigma^{+}=\left\{\alpha \in \Delta-\Delta\left(\pi_{1}\right) \mid \alpha>0\right\}, \Sigma^{-}=\left\{-\alpha \mid \alpha \in \Sigma^{+}\right\}$. Then set $\mathfrak{n}_{1}=$ $\sum_{\alpha \in \Sigma^{-}} \mathfrak{g}_{\alpha}, \mathfrak{n}_{2}=\sum_{\alpha \in \Sigma^{+}} \mathfrak{g}_{\alpha}, \mathfrak{h}=\tilde{\mathfrak{h}}+\sum_{\alpha \in \Delta\left(\pi_{1}\right)} \mathfrak{g}_{\alpha} . \mathfrak{n}_{1}, \mathfrak{n}_{2}, \mathfrak{h}$ are subalgebras of $\mathfrak{g}$. Furthermore if $\alpha \in \Sigma^{+}$(resp. $\alpha \in \Sigma^{-}$) and $\beta \in \Delta\left(\pi_{1}\right)$ and $\alpha+\beta \in \Delta$, then $\alpha+\beta \in \Sigma^{+}$(resp. $\left.\alpha+\beta \in \Sigma^{-}\right)$; thus $\mathfrak{g}=\mathfrak{n}_{1} \oplus \mathfrak{h} \oplus \mathfrak{n}_{2}$ is a decomposition for $\mathfrak{g}$. Furthermore if $\mathfrak{S}_{i}=\tilde{\mathfrak{h}} \oplus \mathfrak{n}_{i}, i=1,2$, then $\mathfrak{n}_{i} \subset\left[\mathfrak{S}_{i}, \mathfrak{S}_{i}\right]$ for $i=1,2$. Thus $\mathfrak{g}=\mathfrak{n}_{1} \oplus \mathfrak{h} \oplus \mathfrak{n}_{2}$ is a triangular decomposition for $\mathrm{g}$. Let $N_{1}, H, N_{2}, K$ be respectively connected subgroups of $G$ corresponding to $\mathfrak{n}_{1}, \mathfrak{h}, \mathfrak{n}_{2}$ and $\mathfrak{h} \oplus \mathfrak{n}_{1}$. Then $K \backslash G$ is the most general Kaehlerian $C$-space. Thus every Kaehlerian $C$-space corresponds to a connected, simply connected, complex semisimple Lie group $G$ with a triangular decomposition $N_{1}, H, N_{2}$. In the following we assume that $G, N_{1}, H, N_{2}, K$ are as above.

It is well known that if $K \backslash G$ is a compact complex manifold then if $W$ is a finite-dimensional holomorphic $H$ module $\operatorname{dim}(\Gamma(W))<\infty$. Thus $\Gamma_{0}(W)=\Gamma(W)$. If $W$ is a simple $H$ module, then by the Corollary to Theorem $4.1, \Gamma(W)$ is a simple finite-dimensional holomorphic $G$ module isomorphic with $W^{*}$ or $\Gamma(W)=\{0\}$ if $\operatorname{dim} W^{*}=\infty$. Thus if $\Gamma(W) \neq\{0\}$ then $\Gamma(W)$ is the finite-dimensional simple $g$ module corresponding to a dominant integral form $\Lambda$ on $\tilde{\mathfrak{h}}$ with respect to $\pi$ (see [3] or [4]). Thus $\Gamma(W)=\sum V_{\lambda}, \lambda \in \tilde{\mathfrak{h}}^{*}$ and $V_{\lambda}=\{v \in \Gamma(W) \mid h \cdot v=\lambda(h) v$ for all $h \in \tilde{\mathfrak{L}}\}$. If $V_{\lambda} \neq\{0\}$ then $\lambda \leqq \Lambda$. And $\operatorname{dim} V_{\Lambda}=1$. Furthermore if $\alpha \in \Delta, \alpha>0$, and $x \in \mathfrak{g}_{\alpha}$, then $x \cdot V_{\Lambda}=0$. Thus $V_{\Lambda} \subset \Gamma(W)^{\mathfrak{n}_{2}}$. Since $\Gamma(W)^{\mathfrak{n}_{2}}$ is irreducible as an $\mathfrak{h}$ module $\left.\Gamma(W)^{\mathfrak{n}_{2}}=U(\dot{i})\right) \cdot V_{\Lambda}$. Let $\left\{h_{1}, \ldots, h_{l}\right\} \subset \tilde{\mathfrak{h}}$ be defined by $\alpha_{i}\left(h_{j}\right)=\delta_{i j}$ where $\pi=\left\{\alpha_{1}, \ldots, \alpha_{l}\right\}$. Set $\tilde{\mathfrak{h}}_{1}=\sum_{\alpha_{i} \in \pi-\pi_{1}} \boldsymbol{C} h_{i}, \tilde{\mathfrak{h}}_{2}=\sum_{\alpha_{i} \in \pi_{1}} C h_{i}$. Then

$$
\tilde{\mathfrak{h}}=\tilde{\mathfrak{h}}_{1} \oplus\left(\tilde{\mathfrak{h}}_{2} \oplus \sum_{\alpha \in \Delta\left(\pi_{1}\right)} \mathfrak{g}_{\alpha}\right)=\tilde{\mathfrak{h}}_{1} \oplus \mathfrak{g}_{1} \text {. }
$$

$\mathfrak{g}_{1}=\tilde{\mathfrak{h}}_{2} \oplus \sum_{\alpha \in \Delta\left(\pi_{1}\right)} \mathfrak{g}_{\alpha}$ and $\mathfrak{g}_{1}$ is complex semisimple. Thus $\Gamma(W)^{\mathfrak{n}_{2}}$ is the simple finite-dimensional module for $\mathfrak{g}_{1}$ with highest weight $\left.\Lambda\right|_{\tilde{f}_{2}}$ with respect to $\pi_{1}$. Let $\sigma$ be the action of $\mathfrak{h}$ on $\Gamma(W)^{\mathfrak{n}_{2}}$; then $\sigma=\left.\Lambda\right|_{\tilde{\mathfrak{h}}_{1}} \otimes \hat{\sigma}$, $\hat{\sigma}$ the action of $\mathfrak{g}_{1}$ on the module $W$ with highest weight $\Lambda \mid \mathfrak{h}_{2}$.

Let $V$ be an arbitrary finite-dimensional simple holomorphic $G$ module. Then $V$ corresponds to a dominant integral form $\Lambda$ on $\tilde{\mathfrak{h}}$ with respect to $\pi$. $V^{\mathfrak{n}_{2}}$ is a nonzero simple holomorphic module for $H$ (resp. $\mathfrak{h}$ ) and $\left(V^{\mathfrak{n}_{2}}\right)^{*}$ is $G$ isomorphic with $V$. Thus by the Corollary to Theorem $4.1 V$ is equivalent to $\Gamma\left(V^{\mathfrak{n}_{2}}\right)$. As before 
$V_{\Lambda} \subset V^{\mathfrak{n}_{2}}$ and thus $V^{\mathfrak{n}_{2}}=U(\mathfrak{h}) \cdot V_{\Lambda}$. And thus $\mathfrak{h}$ acts on $V^{\mathfrak{n}_{2}}$ by the representation $\sigma=\Lambda \tilde{h}_{1} \otimes \hat{\sigma}$ where $\hat{\sigma}$ is the finite-dimensional representation of $g_{1}$ with highest weight $\left.\Lambda\right|_{\tilde{h}_{2}}$ with respect to $\pi_{1}$. We have thus proved

TheOREM 5.1 (BoReL-WeIL). Let $G, \mathfrak{g}, \tilde{\mathfrak{h}}, \Delta, \pi, \pi_{1}, H, K, \tilde{\mathfrak{h}}_{1}, \tilde{\mathfrak{h}}_{2}, \mathfrak{g}_{1}$ be as above. Let $W$ be a finite-dimensional simple holomorphic module for $H$ with action $\sigma$. Then $\Gamma(W)=\{0\}$ if $\sigma=\lambda \otimes \hat{\sigma}$ and there is no dominant integral form on $\tilde{\mathfrak{h}}$ with respect to $\pi$ such that $\lambda=\left.\Lambda\right|_{\mathfrak{h}_{1}}$ and $(\hat{\sigma}, W)$ the $\mathrm{g}_{1}$ module corresponding to the dominant integral form $\left.\Lambda\right|_{\tilde{\mathfrak{H}}_{2}}$ on $\tilde{\mathfrak{h}}_{2}$ with respect to $\pi_{1}$. Conversely if $\sigma=\lambda \otimes \hat{\sigma}$ and there is a dominant integral form $\Lambda$ on $\tilde{\mathfrak{h}}$ with respect to $\pi_{1}$ such that $\lambda=\left.\Lambda\right|_{\mathfrak{h}_{1}}$ and $(\hat{\sigma}, W)$ is the simple $\mathrm{g}_{1}$ module with highest weight $\left.\Lambda\right|_{\tilde{\mathfrak{H}}_{2}}$ with respect to $\pi_{1}$, then $\Gamma(W)$ is $G$ isomorphic with the simple $G$ module with highest weight $\Lambda$ with respect to $\pi$.

The $G$ module $\Gamma(W)$ can be interpreted in the language of cohomology. That is, retaining the notation of Theorem 5.1 , let $\left(E^{\sigma}, \pi\right)$ be the homogeneous vector bundle over $K \backslash G$ (see [1]) induced from $(\sigma, W)$. (That is, let $E^{\prime}=G \times W$ and let $K$ act on $E^{\prime}$ from the left by $h \cdot(g, v)=(h g, h v)$ for $h \in K$ and let $E^{\sigma}=K \backslash E^{\prime}$. Let $\pi[g, v]=K g \in K \backslash G$ where $[g, v]$ is the class of $(g, v)$. Then $\left(E^{\sigma}, \pi\right)$ is the induced vector bundle.) A local holomorphic cross-section of $E^{\sigma}$ is a holomorphic map $f$ of an open subset $U$ of $K \backslash G$ into $E^{\sigma}$ such that for $x \in U, \pi f(x)=x$. Let $\mathscr{S} E^{\sigma}$ be the sheaf of germs of local holomorphic cross-sections of $E^{\sigma}$ and let $H^{p}\left(K \backslash G, \mathscr{S} E^{\sigma}\right)$ be the $p$ th cohomology group of $K \backslash G$ with coefficients in the sheaf $\mathscr{S} E^{\sigma}$. Then $H^{0}\left(K \backslash G, \mathscr{S} E^{\sigma}\right)=\Gamma(W)$.

Thus Theorem 5.1 completely describes $H^{0}\left(K \backslash G, \mathscr{S} E^{\sigma}\right)$ for arbitrary holomorphic finite-dimensional representations $(\sigma, W)$ of $H$. The higher cohomology groups have been studied in Bott [1]. We will study these groups in a later paper.

It is reasonable to ask about the structure of a Lie algebra with triangular decomposition. We will study such algebras in a later paper.

\section{BiBLIOGRAPHY}

1. R. Bott, Homogeneous vector bundles, Ann. of Math. (2) 66 (1957), 203-248.

2. P. P. Želobenko, The theory of linear representations of complex and real Lie groups, Trudy Moskov. Mat. Obšč. 12 (1963), 53-98; English transl., Trans. Moscow Math. Soc. 1963, 57-110.

3. Séminaire, "Sophus Lie," Mimeographed Notes, École Norm. Sup. Paris, 1955.

4. N. Jacobson, Lie algebras, Interscience, New York, 1962.

5. H. C. Wang, Closed manifolds with homogeneous complex structure, Amer. J. Math. 76 (1954), 1-32.

University of CALIFORNIA, Berkeley, California 